\title{
Calidad de las instituciones de educación superior desde la perspectiva de América Latina y el Caribe*
}

[Artículos de Reflexión, Reseñas y Controversias]

\author{
Dora Ligia Páez Luna ${ }^{* *}$ \\ Diana Rocío Camargo Celis ${ }^{* * *}$ \\ Yasser de Jesús Muriel-Perea ${ }^{* * * *}$
}

Recibido: O7 de junio de 2020

Revisado: 27 de junio de 2020

Aceptado: 15 de septiembre de 2020

\footnotetext{
* Artículo resultado de reflexión.

** Universidad Santo Tomás. Magíster en Educación. Especialista en el Enfoque para el Desarrollo de Competencias. Licenciada en Ciencias de la Educación-Filosofía-Historia. Correo electrónico: doralipaez@yahoo.es. ORCID: https://orcid.org/0000-0002-23186843. CVLAC:

https://scienti.minciencias.gov.co/cvlac/visualizador/generarCurriculoCv.do?cod_rh $=0$ $\underline{001458360}$

*** Universidad Santo Tomás. Magíster en Educación. Licenciada en Filosofía e Historia. Correo electrónico: dianacamargo@ustadistancia.edu.co. ORCID:

https://orcid.org/0000-0001-5615-9676. CvLAC:

https://scienti.minciencias.gov.co/cvlac/visualizador/generarCurriculoCv.do?cod_rh $=0$ 000069505\#

${ }_{* * * *}$ Universidad Santo Tomás, Colombia. Magíster en Ingeniería. Magíster en Dirección y Administración de Empresas. Especialista en Docencia Universitaria. Ingeniero de sistemas. Correo electrónico: yassermuriel@ustadistancia.edu.co. ORCID: https://orcid.org/0000-0001-6304-5634. CvLAC:

https://scienti.minciencias.gov.co/cvlac/visualizador/generarCurriculoCv.do?cod_rh $=0$ 000585360.
} 
Cómo citar este artículo:

Páez Luna, D. L., Camargo Celis, D. R. y Muriel Perea, Y. J. (2020). Calidad de las instituciones de educación superior desde la perspectiva de América Latina y el Caribe. Signos, Investigación en sistemas de gestión, 13(1).

https://doi.org/10.15332/24631140.6350

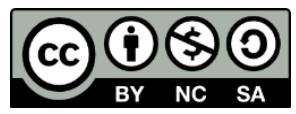

\section{Resumen}

La calidad educativa conlleva la formación integral de ciudadanos y, por tanto, es fundamental para el desarrollo de las personas y los países. El sector educativo está llamado a responder con pertinencia y calidad a las demandas sociales y económicas de los países de América Latina y el Caribe. El objetivo de este artículo es analizar el concepto de calidad desde la perspectiva de seis países de esta región para identificar cómo funcionan sus sistemas de aseguramiento de la calidad y establecer similitudes y diferencias. Se utilizó el estudio de caso y el análisis documental como técnicas de recolección de información. El criterio para seleccionar los casos fue pertenecer a la Red Iberoamericana para el Aseguramiento de la Calidad de la Educación Superior (RIACES) y tener posicionamiento en los rankings. Los resultados muestran que a partir de la década de 1990 se fortalecieron más las políticas de mejoramiento de la calidad en las instituciones de educación superior de la región. Se concluyó que, a pesar de los avances logrados, aún persiste un gran desafío en la integración regional de los sistemas, la respuesta a las necesidades del contexto y la medición del impacto.

Palabras clave: instituciones de educación superior, aseguramiento de la calidad, calidad, América Latina y el Caribe, rankings en educación superior. 


\section{Quality of higher education institutions from the Latin America and the Caribbean perspective}

\section{Abstract}

The quality of education entails the integral formation of citizens and, therefore, it is paramount for people and countries development. The education sector is bound to adequately respond with quality to the social and economic demands of Latin America and the Caribbean countries. This article aims at analyzing the concept of quality from the perspective of six countries in that region to identify how their quality assurance systems work and for establishing both similarities and differences. The case study and documentary analysis were used as information gathering techniques. The cases selecting criteria was to belong to the IberoAmerican Network for Quality Assurance in Higher Education (RIACES) and to be positioned in the rankings. The results show that starting in the 1990s, quality improvement policies in the region's higher education institutions were further strengthened. It was concluded that, despite the progress made, there is still a great challenge in the regional integration of systems, the response to the context needs and the impact measurement.

Keywords: higher education institutions, quality assurance, quality, Latin America and the Caribbean, higher education rankings.

\section{A qualidade das instituições de ensino superior sob a perspectiva da América Latina e do Caribe}

\section{Resumo}

A qualidade da educação pressupõe a formação integral dos cidadãos e, portanto, é fundamental para o desenvolvimento das pessoas e dos países. O setor educacional é chamado a responder com relevância e qualidade às demandas sociais e econômicas dos países da América Latina e do Caribe. O objetivo deste artigo é analisar o conceito de 
qualidade da perspectiva de seis países desta região para identificar o funcionamento dos seus sistemas de garantia de qualidade e para estabelecer semelhanças e diferenças. O caso de estudo e a análise documental foram utilizados como técnicas de coleta de informações. O critério de seleção dos casos foi pertencer à Rede Ibero-Americana de Garantia de Qualidade no Ensino Superior (RIACES) e estar posicionado no ranking. Os resultados mostram que as políticas de melhoria da qualidade nas instituições de ensino superior da região foram reforçadas ainda mais desde a década de 1990. Concluiu-se que, apesar dos progressos alcançados, ainda há um grande desafio na integração regional dos sistemas, na resposta às necessidades do contexto e na medição do impacto.

Palavras-chave: instituições de ensino superior, garantia de qualidade, qualidade, América Latina e Caribe, rankings no ensino superior.

\section{Introducción}

El término calidad tiene distintos significados para diferentes personas, entre ellas excepción, perfección, logro de los objetivos, eficiencia, transformación y valor agregado (Harvey y Green, 1993). La primera agencia de acreditación en educación se creó en Estados Unidos en 1885 y su propósito era estandarizar los criterios y currículos de las diferentes universidades con el fin de realizar homologaciones entre ellas (Roa, 2014).

En América Latina y el Caribe, el camino hacia la calidad se inició en los años 6o. Al comienzo hubo resistencia a la evaluación, primero, por la falta de cultura de rendición de cuentas y, luego, por la falta de experiencia en la construcción de procedimientos de evaluación. Posteriormente, el debate sobre la calidad pasó a un nivel superior, ya no sobre la necesidad de la evaluación, sino sobre cómo hacerla de forma más efectiva. 
En la región, la calidad, la evaluación y la acreditación están en crecimiento, debido a la necesidad de mejorar continuamente los procesos en los que se soporta la gestión educativa en cada una de las instituciones de educación superior (IES). Esta mejora es condición ineludible para el posicionamiento de las instituciones en el ámbito educativo a nivel nacional y mundial, y se evidencia en el sistema de rankings, en donde se clasifican las IES y reciben legitimidad y reconocimiento.

El propósito de este artículo es analizar el concepto de calidad desde la perspectiva de seis países de América Latina y el Caribe, para identificar cómo funcionan sus sistemas de aseguramiento de la calidad y establecer similitudes, diferencias y aspectos relevantes. El ejercicio de correlación planteado permite una evaluación a partir de la comparación entre los sistemas de calidad implementados por estos países, con el propósito de determinar el estado actual de estos sistemas en la región. Esto es esencial a la hora de implementar procedimientos que aseguren la calidad de las IES, sobre la base de una información actualizada y pertinente que nutra las decisiones institucionales y garantice una oferta de calidad.

\section{Conceptos de calidad}

La calidad educativa se relaciona con la formación integral de ciudadanos, "dotados de los conocimientos, técnicas y valores básicos y esenciales al ejercicio crítico y creativo de la ciudadanía, al desarrollo de la sociedad, al enriquecimiento del proyecto de expansión de las libertades humanas" (Dias, 2008, p. 26). El concepto de calidad es polisémico; no obstante, hay cierto consenso en que una IES es de calidad si logra sus objetivos propuestos y si impacta de manera positiva a sus estudiantes (Astin, 1995). Igualmente, "una aproximación que tiende a generalizarse cada vez más es la de los rankings internacionales" (Montoya y Escallón, 2013, p. 380) como medidores de la calidad de las IES. 
Históricamente, el concepto de calidad ha sido analizado desde diferentes perspectivas como la suma de características de un producto o servicio que permiten cumplir con los estándares establecidos (Asiyai, 2020). Etimológicamente, el concepto de calidad tiene sus raíces en el vocablo latino qualitas, que equivale a 'cualidad o atributo de algo'. Aunque las características del concepto de calidad son múltiples, para efectos prácticos del presente artículo se tratarán dos:

- Polisémico: son múltiples los significados de este concepto según el ámbito y los fines desde donde se aborde. La complejidad que representa dificulta llegar a una unidad de criterio frente a su definición desde el ámbito estrictamente educativo.

- $\quad$ Sistémico: corresponde a un tratamiento holístico de la calidad en alusión a una relación interdependiente entre todos sus componentes.

Esta acción articuladora con enfoque sistémico implica la amalgama entre eficiencia, eficacia y equidad, como lo propone la Organización de Estados Iberoamericanos (Vaillant y Rodríguez, 2018). Estas características ubican el concepto de calidad en el foco de preocupación de las IES, en donde obviar alguno de estos aspectos puede desestimar y desviar el sentido y los fines de la calidad educativa, de ahí la importancia de preguntarse y tener claridad sobre el para qué y el por qué, más que sobre los cómo de un sistema de aseguramiento de la calidad.

En la tabla 1, se presentan algunos de los conceptos de calidad más representativos en América Latina y el Caribe, así como sus similitudes y diferencias, a partir del análisis del texto de González y Espinoza (2008) y los conceptos de calidad del Consejo Nacional de Acreditación (CNA) y de la Comisión Nacional de Aseguramiento de la Calidad de la Educación Superior (Conaces) en Colombia. 
Tabla 1. Conceptos de calidad

\begin{tabular}{l|l|}
\multicolumn{1}{c|}{ Referentes } & \multicolumn{1}{c}{ Calidad } \\
$\begin{array}{l}\text { González y } \\
\text { Espinoza } \\
\text { (2008) }\end{array}$ & $\begin{array}{l}\text { Perfección, consistencia, cero defectos, medible, hacer las cosas bien } \\
\text { Aptitud para el logro de un propósito, de una misión. Acomodación de un } \\
\text { producto o servicio según especificaciones del cliente. }\end{array}$ \\
& $\begin{array}{l}\text { Como transformación, cuestiona la calidad centrada en el producto } \\
\text { (Eliot,1992). Debe estar centrada en el estudiante, empoderarlo de su } \\
\text { proceso de aprendizaje, con el fin de que realice una autotransformación. }\end{array}$ \\
\hline Dias (2008) & $\begin{array}{l}\text { Construcción social que varía según los intereses del grupo dentro y fuera } \\
\text { de la institución así: } \\
\text { Académicos: conocimientos y saberes } \\
\text { Empleadores: competencias de los estudiantes } \\
\text { Estudiantes: empleabilidad }\end{array}$
\end{tabular}

Fuente: elaboración propia.

\section{Metodología}

Para cumplir el objetivo de la investigación, se utilizó el método de investigación cualitativa de estudio de caso y el análisis documental como técnica de recolección de información. Según Flyvbjerg (2004), el método de estudio de caso permitió conocer cómo se entiende el concepto de calidad en los contextos particulares de los países de América Latina y el Caribe, ya que "la ciencia social no ha logrado producir una teoría general, independiente del contexto, y, por lo tanto, nada tiene que ofrecer en última instancia salvo un conocimiento concreto y dependiente del contexto" (p. 36).

Para seleccionar los casos estudiados, se tuvo en cuenta que "esta tarea inicial consiste en considerar qué población es relevante para el foco de la investigación que se va a desarrollar" (Johnson y Christensen, 2014, p. 369); por tanto, se utilizó la técnica que LeCompte et ál. (1993) denominan selección basada en criterios. El criterio utilizado para seleccionar los países de América Latina y el Caribe a estudiar, con base en la relevancia de la información que puedan aportar, fue pertenecer a RIACES, tener 
posicionamiento en los rankings y poseer acceso a la información. Los países seleccionados fueron Argentina, Cuba, Colombia, Brasil, Chile y México.

Como técnica de recolección de información, se utilizó el análisis documental con el fin de identificar cómo se entiende la calidad en cada país, sus similitudes y sus diferencias. El análisis se centró en identificar la legislación, los lineamientos del sistema de aseguramiento de la calidad y las estadísticas relevantes del sistema educativo. En la legislación se pudo apreciar el interés constante por la alta calidad en cada país y la obligatoriedad del mismo. En los lineamientos se observaron qué aspectos de la calidad se privilegian, mientras que en las estadísticas se constató cuántas tienen reconocimiento de alta calidad de las IES de cada país.

Las lecturas de los documentos se abordan mediante análisis de contenido. Para acceder a los documentos se consultaron bases de datos como Dialnet, Scopus y Vlex. Los principales documentos analizados por país, además de la información del QS Latin American University Rankings (2020) y el Academic Ranking of World Universities (2019), se muestran en la tabla 2.

Tabla 2. Normas y lineamientos analizados

\begin{tabular}{ll}
\multicolumn{1}{c}{ País } & \multicolumn{1}{c}{ Documentos } \\
\hline Argentina & $\begin{array}{l}\text { Ley de Educación Superior 24521 de julio de } 1995 \\
\text { Resolución Ministerial 31/2005, evaluación externa }\end{array}$ \\
\hline Cuba & $\begin{array}{l}\text { Resolución } 134 \text { del } 6 \text { de julio del 2014, creación Junta de Acreditación } \\
\text { Nacional } \\
\text { Resolución 31 de 2005, Sistema de Evaluación y Acreditación de Carreras } \\
\text { Universitarias }\end{array}$ \\
\hline Colombia & $\begin{array}{l}\text { Ley 30 de 1992, Educación Superior } \\
\text { Ley General de Educación 115 de 1994, lineamientos de acreditación } \\
\text { institucional } \\
\text { Decreto 1330 de 2019 }\end{array}$ \\
\end{tabular}

SIGNOS, Investigación en Sistemas de Gestión

ISSN: 2145-1389 | e-ISSN: 2463-1140 | DOI: https://doi.org/10.15332/24631140

Vol. 13 N. ${ }^{\circ} 1$ | enero-junio de 2021 


\begin{tabular}{|l|l|}
\multicolumn{1}{c|}{ País } & \multicolumn{1}{c}{ Documentos } \\
Brasil & $\begin{array}{l}\text { Ley 10861 de 2004, Sistema Nacional de la Evaluación de la Educación } \\
\text { Superior (Sinaes) }\end{array}$ \\
\hline Chile & $\begin{array}{l}\text { Ley 20129 de 2006, Sistema Nacional para el Aseguramiento de la Calidad de } \\
\text { la Educación Superior (Sinaces) } \\
\text { Ley } 21091 \text { de1 } 29 \text { de mayo de 2018, modificación al Sinaces }\end{array}$ \\
\hline México & Ley para la Coordinación de la Educación Superior, diciembre 29 de 1978
\end{tabular}

Fuente: elaboración propia.

\section{Análisis de la información}

De acuerdo con la metodología establecida, se identificaron las perspectivas del concepto de calidad adoptadas por los países estudiados.

\section{Sistema de aseguramiento de la calidad en Argentina}

En Argentina, la Comisión Nacional de Evaluación y Acreditación Universitaria (Coneau), integrada por doce miembros, es el organismo encargado del Sistema de Evaluación de la Calidad de los Posgrados. Mediante la Ley de Educación Superior 24521 de julio de 1995, se estableció el carácter obligatorio de la acreditación de los programas de posgrado. La reglamentación y los estándares de este proceso se formalizaron mediante la Resolución 160 del 29 de diciembre de 2011, expedida por el Ministerio de Educación.

Esta misma ley adopta tres tipos de posgrados: especializaciones, maestrías y doctorados. Los aspectos evaluados relacionados con el plan de estudios, la modalidad de formación y la carga horaria se establecen en la Resolución 160/11 del Ministerio de Educación.

La Coneau programa dos veces al año el proceso de acreditación, tanto para los programas en funcionamiento como para las nuevas propuestas. Este proceso se organiza para todas las áreas del conocimiento y se lleva a cabo mediante etapas, según las diferentes áreas disciplinares existentes. 
De acuerdo con el informe de la Coneau, Argentina cuenta con 444 doctorados, 1041 programas de maestría y 1665 especializaciones, acreditados por el término de tres años (Coneau, 2019).

\section{Sistema de aseguramiento de la calidad en Cuba}

Cuba cuenta con dos sistemas. El primero es el Sistema Universitario de Programas de Acreditación (Supra), como órgano encargado del aseguramiento de la calidad de la educación cubana. Su órgano directivo es la Junta de Acreditación Nacional (JAN), que es aprobada mediante la Resolución 24 del 2014. Esta norma determina los documentos básicos para la operación del sistema: el reglamento, el patrón de calidad y la guía de evaluación, que se crea mediante la Resolución 134 del 6 de julio de 2004.

Se determinan tres niveles de acreditación para los programas de educación superior: carrera autorizada, certificada y de excelencia. El primer nivel es de carácter obligatorio; es el requisito previo para el funcionamiento de toda IES. Así mismo, este nivel se constituye en la base sobre la cual se funda el mejoramiento continuo de la calidad del sistema educativo cubano, a partir de su fase inicial. Los otros dos niveles son opcionales; en caso de asumirlos, sus requisitos previos son una autoevaluación y una evaluación externa.

Según Horruitiner (2007), "las carreras acreditadas con el nivel de excelencia [...] son 15 en la Universidad de La Habana, 8 en el Instituto Superior Politécnico José Antonio Echeverría y 4 en la Universidad de Oriente" (p. 11). Así mismo, los niveles de acreditación para las maestrías en Cuba (Organización de Estados Iberoamericanos, 2005) son 238 autorizadas, 21 en el nivel de excelencia, 48 certificadas y 10 ratificadas. Estas cifras evidencian el interés del Ministerio de Educación cubano por 
ampliar de manera creciente el rango de cobertura y de calidad de la educación superior.

\section{Sistema de aseguramiento de la calidad en Colombia}

En Colombia, los principales mecanismos para el aseguramiento de la calidad de la educación superior son la acreditación de alta calidad de instituciones y programas, y el registro calificado de programas. La Ley 30 de 1992, que es la norma rectora de la educación superior en el país, estableció el Sistema Nacional de Acreditación con el objetivo principal de "garantizar a la sociedad que las instituciones de educación superior que hacen parte del sistema cumplen con los más altos requisitos de calidad y que realizan sus propósitos y objetivos" (art. 53). Esta ley dispone que la acreditación de alta calidad es voluntaria. En 1994 surgió la Ley General de Educación, que regula el servicio público de educación superior (Congreso de la República de Colombia, 2020).

La Ley 1188 de 2008 regula el registro calificado e indica que es "el instrumento del Sistema de Aseguramiento de la Calidad de la Educación Superior mediante el cual el Estado verifica el cumplimiento de las condiciones de calidad por parte de las instituciones de educación superior" (art. 1). Esta ley está hoy reglamentada por el Decreto 1330 de 2019.

El sistema de aseguramiento de la calidad en Colombia es progresivo. Primero, se obtiene el registro calificado, que es obligatorio y habilitante para recibir estudiantes nuevos. Este registro tiene una vigencia de siete años para los programas que lo obtienen. En segundo lugar, se puede obtener la acreditación de alta calidad, que, por su carácter voluntario y sus características, es el mayor reconocimiento de calidad de una IES en Colombia. 
Los organismos principales del sistema son: el Consejo Nacional de Educación Superior (CESU), el CNA y el Conaces. El CESU es un organismo vinculado al Ministerio Nacional de Educación en Colombia (MEN) y se encarga de asesorarlo en la definición de políticas de educación superior. El CNA fue creado en 1995 y se encarga de fomentar la acreditación de las IES en Colombia. El Conaces, por su parte, se encarga de emitir recomendaciones al MEN sobre el otorgamiento o no del registro calificado para los programas académicos que lo solicitan.

De acuerdo con el Sistema Nacional de Información de la Educación Superior (SNIES, 2020), en Colombia hay 299 IES, de las cuales 66 cuentan con acreditación de alta calidad. Esta cifra equivale al $22 \%$ de las IES del país (figura 1).

Figura 1. Porcentaje de IES acreditadas y no acreditadas

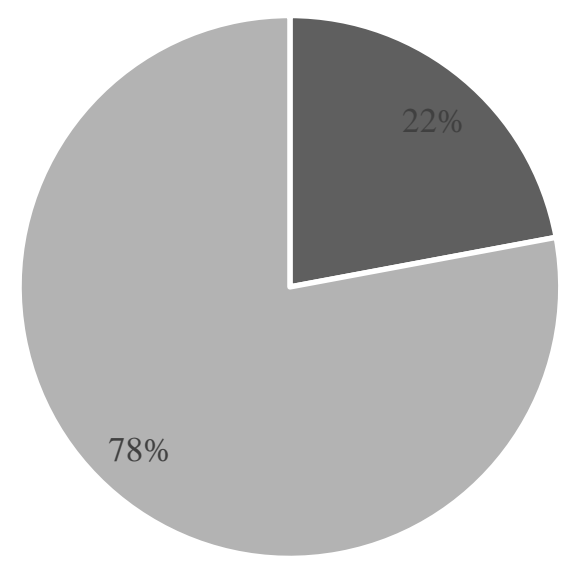

- Acreditadas - No acreditadas

Fuente: elaboración propia. 
En total, hay 13594 programas, todos con registro calificado dada la obligatoriedad que establece la norma. La distribución por niveles de formación se observa en la figura 2.

Figura 2. Cantidad de programas por nivel

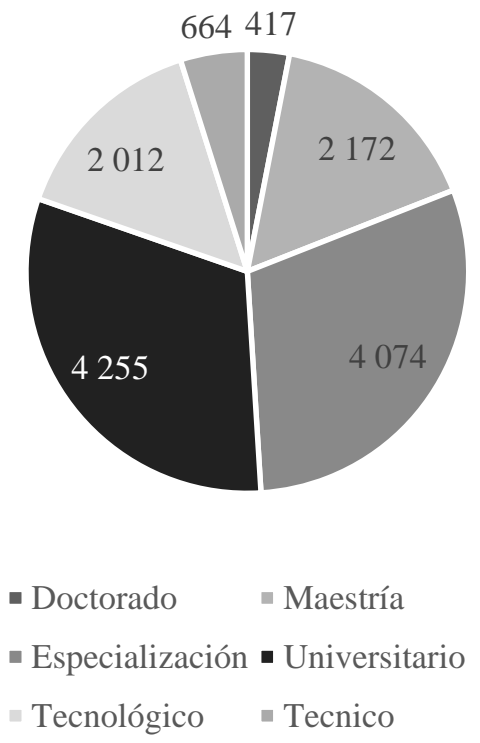

Fuente: elaboración propia.

\section{Sistema de aseguramiento de la calidad en Brasil}

En Brasil, la Ley 10861 de 2004 creó el Sistema Nacional de Avaliação da Educação Superior (Sinaes). Este organismo se encarga de asegurar el proceso de evaluación del desempeño de las IES, los programas de pregrado y los estudiantes (Sinaes, 2020). La evaluación de alta calidad de los programas de posgrado es realizada por la Fundación de Coordinación para el Mejoramiento del Personal de Educación Superior (Capes). Al igual que otros países, en Brasil existe un Consejo Nacional de Educación, el cual brinda recomendaciones en materia de política educativa al Ministerio de Educación Nacional. 
Los procesos de evaluación son coordinados por la Comisión Nacional de Evaluación de la Educación Superior (Conaes). Esta entidad establece las directrices de las evaluaciones de las instituciones y los programas, mientras que su ejecución está a cargo del Instituto Nacional de Estudios e Investigaciones Educativas (INEP) (Trindade y Sotomayor, 2007). Las instituciones se evalúan en una escala de 1 a 5 . Los resultados de las evaluaciones permiten obtener una visión de la calidad de las IES y de los programas (INEP, 2020).

Para el caso de las instituciones y los programas académicos, "el Sinaes realiza la acreditación institucional que incluye el proceso de autoevaluación, la posterior evaluación externa y la certificación. Asimismo, la evaluación de programas se realiza a través del análisis de las condiciones de enseñanza y de los recursos humanos, materiales y didácticos (González, 2005, p. 5). En el caso de los estudiantes, el sistema reúne información del examen nacional de desempeño de estudiantes. En Brasil, las IES se clasifican en universidades, institutos federales de educación, ciencia y tecnología, centros universitarios y facultades. Las universidades son consideradas de mejor calidad, en parte porque hacen investigación y poseen profesores con dedicación de tiempo completo (Barreyro y Costa, 2015). En Brasil existen 3146 IES. De este número, 205 tienen carácter de universidad, 84 tienen evaluación en nivel 4 y 5 en el Sinaes (E-MEC Brasil, 2020), lo cual equivale al $41 \%$ de las universidades (figura 3). 


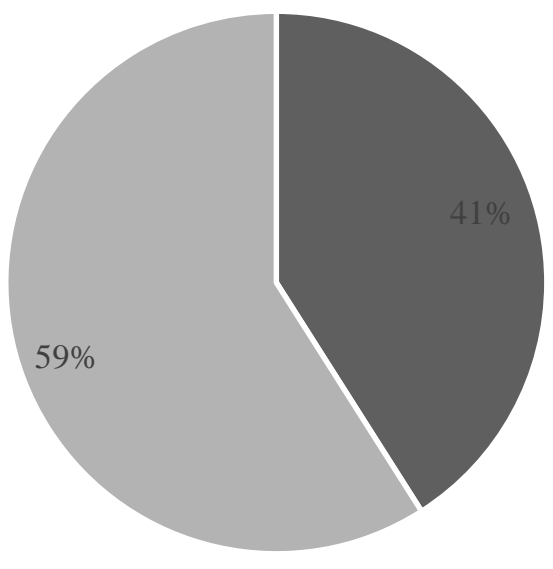

- Calificación 4 o 5 - Calificación 3 o menos

Fuente: elaboración propia.

\section{Sistema de aseguramiento de la calidad en Chile}

En Chile, el Sistema Nacional para el Aseguramiento de la Calidad de la Educación Superior (Sinaces) fue formalizado a través de la Ley 20129 del Ministerio de Educación (2006), pero recientemente modificado por la Ley 21091 de1 29 de mayo de (2018). Su propósito es preservar y promover la calidad de la educación superior, por medio de la autorización para el funcionamiento a nuevas instituciones, la acreditación institucional de carreras y programas de posgrado, así como la provisión de información pública.

El sistema está integrado por el Ministerio de Educación, órgano rector representado por la Subsecretaría de Educación Superior, el Consejo Nacional de Educación (CNED), la Comisión Nacional de Acreditación (CNA) y la Superintendencia de Educación Superior, que conforman el comité coordinador. Sesionan al menos seis veces al año, aunque se 
reúnen extraordinariamente por solicitud de cualquiera de sus miembros o por petición de los estamentos que lo integran. Asimismo, en su quehacer hacen parte las IES.

A través del CNED, Chile es miembro desde el 21 de mayo de 1995 de la Red Internacional de Agencias de Aseguramiento de la Calidad en la Educación Superior (INQAAHE, por su sigla en inglés), una asociación de instituciones que junto a organismos académicos nacionales como agencias acreditadoras trabaja en pro del aseguramiento de la calidad en la educación superior a nivel internacional. De acuerdo con el artículo 1 de la la Ley 21091 de 2018, a los organismos públicos mencionados les corresponde:

- La implementación de políticas que promuevan la calidad en las dinámicas propios de las IES.

- La identificación, almacenamiento y divulgación de la información necesaria para la gestión del sistema.

- $\quad$ El licenciamiento de instituciones nuevas de educación superior a través del CNED.

- La acreditación de las IES autónomas y la acreditación de carreras o programas de pregrado y posgrado.

- La fiscalización del cumplimiento de los compromisos financieros, administrativos y académicos de las IES, así como la verificación del acatamiento de las normas para la consecución de sus fines.

Las universidades, los institutos profesionales y los centros de formación técnica autónomos pueden someterse voluntariamente a acreditación institucional, excepto los programas de pregrado en Medicina, Odontología y todas las pedagogías, así como los programas de doctorado y las especialidades del área de la salud, para quienes la acreditación es obligatoria. Actualmente, Chile cuenta con 41 IES acreditadas entre 
públicas y privadas. De igual forma, las IES deben incluir información en los medios de divulgación que utilicen sobre su proceso de acreditación institucional, estado y resultados.

\section{Sistema de aseguramiento de la calidad en México}

Según la Organización para la Cooperación y el Desarrollo Económicos (2019), "el sistema de educación superior mexicano, con 13 subsistemas, es altamente complejo y diverso" (p. 9), diferente de otros países en la región. En primer lugar, porque el contexto social y político ha permeado los esfuerzos que desde los años 70 viene haciendo el Estado con el propósito de erradicar la baja calidad y orientar a las instituciones hacia el mejoramiento de sus funciones. El 29 de diciembre de 1978 se creó la Ley para la Coordinación de la Educación Superior y, posteriormente, el Sistema Nacional de Planeación Permanente de Educación Superior (Sinappes) con sus correspondientes instancias (Buendía, 2011).

En segundo lugar, porque en este sistema operan políticas diferenciadas, por una parte, las que aplican para la educación tecnológica y para la educación superior universitaria y son definidas por la Secretaría de Educación Pública (SEP), ente encargado del gabinete presidencial de "crear condiciones que permitan asegurar el acceso, de las mexicanas y mexicanos, a una educación de excelencia con equidad, universalidad e integralidad, en el nivel y modalidad que la requieran y en el lugar donde la demanden" (SEP, 2020). Por otra parte, se encuentran las políticas que corresponden a los programas de posgrado establecidas por el Consejo Nacional de Ciencia y Tecnología (Conacyt).

Un antecedente en el camino hacia el aseguramiento de la calidad es la institucionalización de la Evaluación de la Educación Superior (19891994), gracias a los acercamientos entre el Gobierno federal y la Asociación Nacional de Universidades e Instituciones de Educación Superior 
(Anuies), por medio de la Comisión Nacional de Evaluación (Conaeva). Se aplicó la estrategia y operación del Sistema Nacional de Evaluación "a partir de tres líneas de acción: la autoevaluación de las instituciones, la evaluación del sistema y los subsistemas y la evaluación interinstitucional de programas académicos y mediante el mecanismo de evaluación de pares calificados" (Buendía, 2011, p. 4), cuyos resultados permitieron a la SEP implementar programas de mejoramiento en diferentes aspectos según los hallazgos, así como destinar presupuesto para su desarrollo. Frente al aseguramiento de la calidad en México operan la autoevaluación institucional, que posibilita el ingreso o el acceso a la Anuies, y la acreditación institucional, a cargo de la Federación de Instituciones Mexicanas Particulares de Educación Superior (Fimpes).

\section{Posición de IES latinoamericanas en los rankings mundiales}

Las clasificaciones académicas de universidades o rankings internacionales de instituciones de educación superior son listados ordenados de universidades que han sido clasificadas de acuerdo con un análisis sobre una serie de criterios y factores, total o parcialmente académicos. Son realizados por grupos de especialistas de organizaciones independientes y "muestran ser una fuente útil de información y una forma de contribuir a la visibilidad y reputación institucional" (BustosGonzález, 2019, p. 1).

En este trabajo se revisó el posicionamiento de los países estudiados a partir de los listados publicados en los Rankings de Shanghái y el QS World Ranking de 2019, por ser dos de las clasificaciones más influyentes. El Ranking Académico de Universidades del Mundo (ARWU, por su sigla en inglés) se dio a conocer en 2003 por el Centro de Universidades de Clase Mundial (CWCU), de la Escuela de Graduados de Educación de la 
Universidad Jiao Tong de Shanghái, China. Este ranking tiene en cuenta aspectos tanto de calidad académica como de investigación. Sus indicadores y ponderaciones para el análisis son la cantidad de distinguidos con el Premio Nobel o la Medalla Fields formados en la universidad (10 \%) o trabajando en la misma (20 \%); el número de investigadores citados en 21 temas generales (20 \%); el número de artículos publicados en las revistas científicas Science y Nature (20 \%); el número de trabajos académicos registrados en los índices del Science Citation Index y el Social Science Citation Index (20 \%), y la producción per cápita, es decir, la puntuación de todos los indicadores anteriores dividida entre el número de académicos a tiempo completo (10 \%). Las IES de los países estudiados e América Latina y el Caribe tienen presencia en el ranking ARWU versión 2020. La totalidad de IES en el ranking por países se observa en la tabla 3 . Brasil cuenta con 23 IES en el ranking, se muestran las cuatro primeras. 
Tabla 3. Instituciones de educación superior de América Latina y el Caribe en el Ranking de Shanghái

\begin{tabular}{|c|c|c|c|c|c|c|c|c|c|c|c|}
\hline \multirow{2}{*}{ País } & \multirow{2}{*}{ Universidad } & \multicolumn{10}{|c|}{ Rango de clasificación según el ranking de Shanghái } \\
\hline & & 1-100 & 101-200 & 201-300 & 301-400 & 401-500 & $501-600$ & $601-700$ & 701-800 & 801-900 & 901-1000 \\
\hline \multirow[t]{4}{*}{ Brasil (23) } & $\begin{array}{l}\text { 1. Universidad } \\
\text { de São Paulo }\end{array}$ & & $\checkmark$ & & & & & & & & \\
\hline & $\begin{array}{l}2-4 \\
\text { Universidad } \\
\text { Federal de Río } \\
\text { de Janeiro }\end{array}$ & & & & $\checkmark$ & & & & & & \\
\hline & 2-4 UNESP & & & & $\checkmark$ & & & & & & \\
\hline & $\begin{array}{l}2-4 \\
\text { Universidad de } \\
\text { Campinas }\end{array}$ & & & & $\checkmark$ & & & & & & \\
\hline \multirow[t]{2}{*}{ México (2) } & $\begin{array}{l}\text { 1. Universidad } \\
\text { Nacional } \\
\text { Autónoma de } \\
\text { México }\end{array}$ & & & $\checkmark$ & & & & & & & \\
\hline & $\begin{array}{l}\text { 2. Instituto } \\
\text { Politécnico } \\
\text { Nacional }\end{array}$ & & & & & & $\checkmark$ & & & & \\
\hline \multirow[t]{3}{*}{$\begin{array}{l}\text { Argentina } \\
\text { (3) }\end{array}$} & $\begin{array}{l}\text { 1. Universidad } \\
\text { de Buenos } \\
\text { Aires }\end{array}$ & & & $\checkmark$ & & & & & & & \\
\hline & $\begin{array}{l}\text { 2. Universidad } \\
\text { de la Plata }\end{array}$ & & & & & & & & $\checkmark$ & & \\
\hline & $\begin{array}{l}\text { 3. Universidad } \\
\text { de Córdoba }\end{array}$ & & & & & & & & & $\checkmark$ & \\
\hline \multirow[t]{2}{*}{ Chile (5) } & $\begin{array}{l}\text { 1. Universidad } \\
\text { de Chile }\end{array}$ & & & & & $\checkmark$ & & & & & \\
\hline & $\begin{array}{l}\text { 2. Universidad } \\
\text { Católica }\end{array}$ & & & & & & $\checkmark$ & & & & \\
\hline
\end{tabular}

SIGNOS, Investigación en Sistemas de Gestión

ISSN: 2145-1389 | e-ISSN: 2463-1140 | DOI: https://doi.org/10.15332/24631140

Vol. 13 N.o 1 | enero-junio de 2021 


\begin{tabular}{|c|c|c|c|c|c|c|c|c|c|c|c|}
\hline \multirow{2}{*}{ País } & \multirow{2}{*}{ Universidad } & \multicolumn{10}{|c|}{ Rango de clasificación según el ranking de Shanghái } \\
\hline & & 1-100 & 101-200 & 201-300 & 301-400 & 401-500 & 501-600 & $601-700$ & 701-800 & 801-900 & 901-1000 \\
\hline & $\begin{array}{l}\text { 3-4. } \\
\text { Universidad } \\
\text { Andrés Bello }\end{array}$ & & & & & & & & & $\checkmark$ & \\
\hline & $\begin{array}{l}\text { 3-4. } \\
\text { Universidad de } \\
\text { Concepción }\end{array}$ & & & & & & & & & $\checkmark$ & \\
\hline & $\begin{array}{l}\text { 5. Universidad } \\
\text { Técnica } \\
\text { Federico Santa } \\
\text { María }\end{array}$ & & & & & & & & & & $\checkmark$ \\
\hline \multirow[t]{2}{*}{$\begin{array}{l}\text { Colombia } \\
\text { (2) }\end{array}$} & $\begin{array}{l}\text { 1. Universidad } \\
\text { Nacional de } \\
\text { Colombia }\end{array}$ & & & & & & & & & $\checkmark$ & \\
\hline & $\begin{array}{l}\text { 2. Universidad } \\
\text { de los Andes }\end{array}$ & & & & & & & & & & $\checkmark$ \\
\hline
\end{tabular}

Fuente: elaboración propia.

SIGNOS, Investigación en Sistemas de Gestión

ISSN: 2145-1389 | e-ISSN: 2463-1140 | DOI: https://doi.org/10.15332/24631140

Vol. 13 N. 01 | enero-junio de 2021 
El QS Latin American University Rankings es una lista de clasificación producida en el Reino Unido por el grupo Quacquarelli Symonds y analiza las IES a partir de indicadores y ponderaciones así: reputación académica (30 \%), reputación de los empleadores (20 \%), proporción de estudiantes por profesor (10 \%), citaciones por profesor (5\%), artículos por profesor (10 \%), personal docente con doctorado ( $5 \%$ ), impacto en la web (5\%), estudiantes internacionales (2.5\%), profesores internacionales (2.5\%) y redes internacionales de investigación (10 \%).

Las IES de los países estudiados en América Latina y el Caribe tienen presencia en el ranking QS versión 2020. Del ranking QS de las 1000 mejores universidades del mundo de 2020, se tomaron las tres mejor posicionadas de los países estudiados (tabla 4). 
Tabla 4. Instituciones de educación superior de América Latina y el Caribe en ranking mundial QS

\begin{tabular}{|c|c|c|c|c|c|c|c|}
\hline \multirow{2}{*}{$\begin{array}{c}\text { País y número de IES en el } \\
\text { ranking }\end{array}$} & \multirow{2}{*}{ Universidad } & \multicolumn{6}{|c|}{ Ranking QS } \\
\hline & & 1-100 & 101-200 & 201-300 & 301-400 & 401-500 & $501-600$ \\
\hline \multirow{3}{*}{ Brasil (19) } & Universidad de São Paulo* & & 116 & & & & \\
\hline & Universidad Estatal de Campiñas* & & & 214 & & & \\
\hline & Universidad Federal de Río de Janeiro * & & & & 358 & & \\
\hline \multirow{3}{*}{ México (13) } & Universidad Nacional Autónoma de México* & & 103 & & & & \\
\hline & Tecnológico de Monterrey & & 158 & & & & \\
\hline & Universidad Anáhuac México & & & & & & $561-570$ \\
\hline \multirow{3}{*}{ Argentina (13) } & Universidad de Buenos Aires* & 74 & & & & & \\
\hline & Pontificia Universidad Católica Argentina & & & & 344 & & \\
\hline & Universidad de Palermo & & & & 383 & & \\
\hline \multirow{3}{*}{ Chile (11) } & Pontificia Universidad Católica de Chile* & & 127 & & & & \\
\hline & Universidad de Chile* & & 189 & & & & \\
\hline & Universidad de Santiago de Chile & & & & & 489 & \\
\hline \multirow{3}{*}{ Colombia (12) } & Universidad de los Andes* & & & 234 & & & \\
\hline & Universidad Nacional de Colombia* & & & 253 & & & \\
\hline & Pontificia Universidad Javeriana & & & & & 468 & \\
\hline Cuba (1) & Universidad de La Habana & & & & & & $501-510$ \\
\hline
\end{tabular}

* Universidades en los dos rankings.

Fuente: elaboración propia.

SIGNOS, Investigación en Sistemas de Gestión

ISSN: 2145-1389 | e-ISSN: 2463-1140 | DOI: https://doi.org/10.15332/24631140

Vol. 13 N. ${ }^{\circ} 1$ | enero-junio de 2021 
El estudio revela que existe interés de parte de los organismos encargados de fomentar la calidad en las IES de América Latina y el Caribe por hacer parte de redes internacionales de agencias de aseguramiento de la calidad, en particular de la INQAAHE. Esto es importante porque facilita la articulación en aspectos internacionales. No obstante, puede afectar la pertinencia de los sistemas al enfocarse en temas globales y descuidar los problemas del entorno. Esta tensión debe ser gestionada estableciendo un balance entre las necesidades locales y globales.

Los resultados muestran que en los años 90 se fortalecieron los sistemas de aseguramiento de la calidad de América Latina y el Caribe. Los sistemas más sólidos en términos del modelo de acreditación son los de los países pioneros en la definición de normas para el fomento de la calidad en la educación superior, tales como Chile y Colombia. Esto evidencia que la gobernabilidad del sistema de aseguramiento de la calidad es fundamental para el logro de los propósitos.

La revisión muestra que, en el caso de México, donde las iniciativas fueron propuestas por la Anuies y concertadas con el Gobierno federal, la experiencia no ha sido del todo satisfactoria. Esto sugiere que es necesario contar con la participación de los públicos de interés de las IES en la definición de políticas de aseguramiento de la calidad, pero debe haber un ente central que coordine y determine hasta qué punto se consideran o no las iniciativas. De lo contrario, pueden generarse silos y complejidades en el sistema de aseguramiento de la calidad.

De acuerdo con los hallazgos, se considera que la alta tasa de desempleo de cada país no ha sido tenida en cuenta en los procesos de acreditación. La calidad educativa no tiene coherencia con el desempleo; cuando esto ocurre, es necesario revisar el mercado laboral o los programas educativos. Lo esencial de los indicadores de calidad es ir más allá del cumplimiento 
de requisitos. En ese orden de ideas, se comprenderá que la calidad en perspectiva de equidad es una urgencia en América Latina y el Caribe.

\section{Conclusiones}

El carácter polisémico del concepto de calidad implica gran divergencia en la forma en que los diferentes países latinoamericanos la asumen, lo que tiene un efecto poco favorable en su evaluación. Se requiere llegar a un consenso sobre su significado y sentido para los diferentes países de la región, a fin de lograr una medición y comparación en igualdad de condiciones.

En la valoración de la calidad educativa, no es suficiente con la eficiencia, la eficacia y la equidad. Es necesario, además, enfocar la atención hacia los procesos de enseñanza, aprendizaje y evaluación en términos del impacto que estos representan en el desarrollo cognitivo, emocional y actitudinal de los estudiantes. En los países estudiados, los entes gubernamentales son los organismos encargados de fortalecer las políticas educativas, dar directrices y proporcionar mecanismos para el funcionamiento del sistema de aseguramiento de la calidad; por tal razón, deben ser ellos los primeros comprometidos.

La evaluación institucional es el proceso por el que iniciaron las IES. En la actualidad, su tránsito hacia la construcción de una cultura de la calidad educativa se ve robustecida, a diferencia de años anteriores en los que se cuestionaba su pertinencia. Aunque los diferentes países de América Latina están comprometidos con la calidad y han creado leyes, mecanismos y entidades para su aseguramiento, los procesos de integración educativa fuera de las fronteras resultan complejos. La evaluación del sistema de aseguramiento de la calidad no escapa a los rasgos de subjetividad inherentes a todo proceso evaluativo, de ahí la 
importancia de dar precisión sobre el margen de error que pueda presentar, como es el caso de la categorización en el ranking. La evolución en las posiciones del ranking muestra cambios atípicos de un año a otro, lo que manifiesta la falta de claridad y estabilidad en los indicadores de medición. América Latina y el Caribe viene avanzando en su compromiso con la calidad en educación superior y esto se evidencia en el posicionamiento de las IES de cinco países de la región en los rankings internacionales, lo que ha contribuido a la construcción de la marca país.

\section{Referencias}

Academic Ranking of World Universities. (2019). Ranking ARWU. http://www.shanghairanking.com/

Asiyai, R. I. (2020). Best practices for quality assurance in higher education: implications for educational administration. International Journal of Leadership in Education. https://doi.org/10.1080/13603124.2019.1710569

Astin, A. (1985). Achieving educational excellence. Jossey-Bass.

Barreyro, G. B. y Costa, F. (2015). Las políticas de educación superior en Brasil en la primera década del siglo XXI: Algunas evidencias sobre impactos positivos en la equidad. Revista Mexicana de Investigación Educativa, 20(64), 17-46.

Buendía, A. (2011). Aseguramiento de la calidad: políticas públicas y gestión universitaria. Estudio sobre impacto de los procesos de aseguramiento de la calidad (AQ) a partir de información provista por actores relevantes: el caso de seis universidades e. CINDA.

Bustos- González, A. (2019). Tránsito de universidad docente a universidad de investigación. ¿Un problema de información académica, de taxonomías o de rankings universitarios? Profesional de la Información, 28(4).

https://doi.org/10.3145/epi.2019.jul.22

CNA Chile. (2020). Consejo Nacional de Acreditación Chile.

https://www.cnachile.cl/Paginas/Inicio.aspx

SIGNOS, Investigación en Sistemas de Gestión

ISSN: 2145-1389 | e-ISSN: 2463-1140 | DOI: https://doi.org/10.15332/24631140

Vol. 13 N.o 1 | enero-junio de 2021 
Comisión Nacional de Evaluación y Acreditación Universitaria. (2019). Posgrados acreditados de la República de Argentina.

https://www.coneau.gob.ar/archivos/publicaciones/CatalogoPosgrados_2019.pdf

Congreso de la República de Argentina. (1995). Ley 24521. Ley de Educación Superior.

https://www.argentina.gob.ar/normativa/nacional/ley-24521-25394/actualizacion

Congreso de la República de Colombia. (1992, diciembre 28). Ley 30. Por la cual se organiza el servicio público de Educación Superior. Diario Oficial 40700. http://www.suin-juriscol.gov.co/viewDocument.asp?ruta=Leyes/1586969

Congreso de la República de Colombia. (2008, 25 de abril). Ley 1188. Por la cual se regula el registro calificado de programas de educación superior y se dictan otras disposiciones. Diario Oficial 46971.

https://docs.colombia.justia.com/nacionales/leyes/ley-1188-de-2008.pdf

Congreso de la República de Colombia. (2020). Ley 115 de 1994. Por la cual se expide la ley general de educación. Diario Oficial 41214. http://www.suinjuriscol.gov.co/viewDocument.asp?ruta=Leyes/1645150

Congreso de los Estados Unidos Mexicanos. (1978, 29 de diciembre). Ley para la Coordinación de la Educación Superior. Diario Oficial de la Federación. http://www.diputados.gob.mx/LeyesBiblio/pdf/182.pdf

Congreso Nacional de Brasil. (2004). Ley 10861. http://www.planalto.gov.br/ccivil 03/_ato2004-2006/2004/lei/l10.861.htm

Consejo Nacional de Educación. (2020). Educación superior. https://www.cned.cl/educacion-superior

Dias, J. (2008). Calidad, pertinencia y relevancia: relación con el resto del sistema y la sociedad. En A. Gazzola y S. Pires (eds.), Hacia una política regional de Aseguramiento de la calidad en Educación superior para América Latina y el Caribe (pp. 14-55). Unesco - IESALC.

E-MEC Brasil. (2020). Cadastro Nacional de Cursos e Instituições de Educação Superior. http://emec.mec.gov.br/

Flyvbjerg, B. (2004). Cinco malentendidos acerca de la investigación mediante los estudios de caso. Revista Española de Investigaciones Sociológicas, 106(1), 33-62.

SIGNOS, Investigación en Sistemas de Gestión ISSN: 2145-1389 | e-ISSN: 2463-1140 | DOI: https://doi.org/10.15332/24631140 Vol. 13 N. ${ }^{\circ} 1$ | enero-junio de 2021 
González, L. y Espinoza, O. (2008). Calidad en la educación superior: concepto y modelos. Calidad en la Educación, 28, 248-276.

http://dx.doi.org/10.31619/caledu.n28.210

Harvey, L. y Green, D. (1993). Defining quality. Assessment and Evaluation in Higher Education, 18(1), 9-34.

Horruitiner, S. P. (2005). El modelo de acreditación de carreras de la educación superior cubana. Mirador, 36(144), 121-132. http://www.scielo.org.mx/scielo.php?script=sci arttext\&pid=So185$\underline{27602007000400008}$

INEP. (2020). Sinaes. http://inep.gov.br/sinaes

Johnson, B. y Christensen, L. (2014). Educational research: quantitative, qualitative, and mixed approaches $\left(5 \cdot{ }^{\mathrm{a}}\right.$ ed.). Sage Publications.

LeCompte, M., Preissle, E. y Tesch, R. (1993). Ethnography and qualitative design in educational research (2. ${ }^{\mathrm{a}}$ ed.). Academic Press.

Ministerio de Educación de Chile. (2006, 17 de noviembre). Ley $\mathrm{N}^{0}$ 20.129. Sistema Nacional de Aseguramiento de la Calidad de la Educacion Superior. https://www.leychile.cl/Navegar?idNorma=255323

Ministerio de Educación de Chile. (2018, 29 de mayo). Ley 21091. Sobre educación superior.

https://www.leychile.cl/Navegar?idNorma=1118991\&idParte=9917404\&idVersion $=2018-05-29$

Ministerio de Educación de Cuba. (2005). Resolución 31. https://www.mes.gob.cu/es/resoluciones

Ministerio de Educación de Cuba. (2014). Resolución 24. https://www.mes.gob.cu/es/resoluciones

Ministerio de Educación Nacional de Colombia. (2019, julio 25). Decreto 1330 de 2019. Por el cual se sustituye el Capítulo 2 y se suprime el Capítulo 7 del Título 3 de la Parte 5 del Libro 2 del Decreto 1075 de 2015 - Único Reglamentario del Sector Educación. https://www.mineducacion.gov.co/1759/articles387348 archivo pdf.pdf 
Montoya, J. y Escallón, E. (2013). Calidad de la educación superior: ċrecursos, actividades o resultados? En E. Orozco (ed.), La educación superior: retos y perspectivas (pp. 379-417). Uniandes.

Organización para la Cooperación y el Desarrollo Económicos. (2019). Higher education in Mexico: labour market relevance and outcomes, higher education. OECD Publishing. https://doi.org/10.1787/9789264309432-en

Organización de Estados Iberoamericanos. (2005). Estructura y titulaciones de educación superior en Cuba. https://www.oei.es/historico/homologaciones/cuba.pdf

QS Quacquarelli Symonds Limited. (2020). QS Latin American University Rankings 2020. https://www.topuniversities.com/university-rankings/latin-americanuniversity-rankings/2020

Roa, A. (2014). Veinte años de la acreditación en Colombia. En V. Roa y I. Pacheco (eds.), Educación superior en Colombia: doce propuestas para la próxima década. Editorial Universidad del Norte.

Secretaría de Educación Pública. (2020, 21 enero). Visión y misión de la SEP. Gobierno de México. https://www.gob.mx/sep/acciones-y-programas/vision-y-mision-de-lasep

Sinaes. (2020). Sistema Nacional de Evaluación de la Educación Superior. http://inep.gov.br/sinaes

SNIES. (2020). Consulte programas académicos. https://snies.mineducacion.gov.co/consultasnies/programa

Trindade, H. y Sotomayor, W. (2007). Evaluación de la educación superior en Brasil: fundamentos, desafios, institucionalización e imágen pública: 2004-2006. Unesco, Instituto Internacional para la Educación Superior en América Latina y el Caribe.

Vaillant, D. R. y Rodríguez, E. (2018). Perspectivas de Unesco y la OEI sobre la calidad de la educación. En H. Monarca (coord.), Calidad de la educación en Iberoamérica: discursos, políticas y prácticas (pp. 136-154). https://ie.ort.edu.uy/innovaportal/file/73018/1/perspectivas-de-unesco-y-oeivaillant-rodriguez.pdf 